\title{
Does automated breast ultrasound (ABUS) add to breast tomosynthesis (DBT) in assessment of lesions in dense breasts?
}

\author{
Rania Mohamed Hegazy ${ }^{*^{*}}$ (D), Omnia Mokhtar Nada ${ }^{2}$ and Engy A. Ali ${ }^{1}$
}

\begin{abstract}
Background: As mammography has its known limitations in dense breast, additional imaging is usually needed. We aimed to evaluate the role of automated breast ultrasound in addition to tomosynthesis in detection and diagnosis of breast lesions in dense breasts. Seventy patients with dense breasts subjected to full-field digital mammography (FFDM) including digital breast tomosynthesis (DBT) and automated breast ultrasound (ABUS). Both studies were evaluated by two experienced radiologists to assess breast composition, mass characterization, asymmetry, calcification, axillary lymphadenopathy, extent of disease (EOD), skin thickening, retraction, architectural distortion, and BIRADS classification. All breast masses were interpreted as above described and then correlated with final pathological diagnosis.

Results: Study included 70 females presenting with different types of breast lesions. Eighty-two masses were detected: 53 benign ( $n=53 / 82), 29$ malignant $(n=29 / 82)$. Histopathology of the masses was reached by core biopsy $(n=30)$, FNAC $(n=14)$, and excisional biopsy $(n=11)$. The rest of the masses $(n=27 / 82)$ were confirmed by their characteristic sonographic appearances; 20 cases of multiple bilateral anechoic simple cysts, 7 typical fibroadenomas showed stationary course on follow-up. As regards the final BIRADS score given for both modalities, tomosynthesis showed accuracy of $93.1 \%$ in characterization of malignant masses with accuracy of $94.3 \%$ in benign masses, on the other hand automated ultrasound showed 100\% accuracy in characterization of malignant masses with $98.1 \%$ accuracy in benign masses.

Conclusion: Adding ABUS to tomosynthesis has proven a valuable imaging tool for characterization of breast lesions in dense breasts both as screening and diagnostic tool. They proved to be more sensitive and specific than digital mammography alone in showing tissue overlap, tumor characterization, lesion margins, extent, and multiplicity of malignant lesions.
\end{abstract}

Keywords: ABUS, DBT, Tomosynthesis

\section{Background}

Although mammography is the main pillar of breast cancer screening programs and is leading to improved treatment options and reduced mortality, yet sensitivity is reduced in populations with dense breast tissue [1]. Performing a supplemental breast ultrasound in women

\footnotetext{
* Correspondence: raniahegazy@hotmail.com; hegazyrania@gmail.com ${ }^{1}$ Clinical and Interventional Radiology, faculty of Medicine, Cairo University, Cairo, Egypt

Full list of author information is available at the end of the article
}

with ACR density $\mathrm{C}$ or $\mathrm{D}$ breast tissue detects an additional approximately $3.5 / 1000$ malignancies occult to mammography. Both mammography and ultrasound have undergone continued development in the last few years [2].

Digital breast tomosynthesis (DBT), a 3D procedure using low-dose digital $\mathrm{X}$-ray projections, increase sensitivity, and specificity of breast cancer detection compared to 2D mammography [3]. 
Automated 3D-ultrasound imaging of the whole breast (AWBU) has the potential to overcome the examinerdependency of handheld ultrasound (HHUS) and to reduce the time of examination and interpretation $[4,5]$. The aim of our study was to evaluate the role of automated breast ultrasound (ABUS) in addition to tomosynthesis in detection and diagnosis of different dense breast lesions and for early detection of breast cancer.

\section{Methods}

This was a retrospective study that included 82 breast masses in 70 female patients detected on mammography and ultrasound. This study was approved by the local Research and Ethical committee; all the included cases gave informed consent.

The study was conducted on 70 patients presented to the breast imaging unit with either palpable breast mass, breast pain, nipple discharge, or for routine check-up. They presented during the period from April 2017 to September 2019. Their ages ranged from 25 to 82 years (mean age, 45 years \pm 9 ). Heterogeneously dense parenchyma (ACR C) or extremely dense breast (ACR D) was included in the study.

Inclusion criteria: Cases with dense breast in mammography (ACR class C and D) who agreed to the study protocol and ABUS study.

Exclusion criteria: Patients who has any contraindication to mammography or cannot lie supine during the ABUS study.

All the cases $(n=70)$ were subjected to both tomosynthesis and automated breast ultrasound. They were asked to expose the upper part of the body. Pregnancy test was required for those within the childbearing period who might be pregnant.

\section{a) Tomosynthesis examination protocol design}

A craniocaudal (CC) and a medio-lateral oblique (MLO) view DBT were obtained with the patient in a standing position using a DBT unit prototype. Breast compression was applied, like that of mammography. Images were acquired with a GE mammography system "GE Senographe Essential with Seno Bright upgrade."

\section{b) Automated breast examination protocol design}

A layer of gel was applied to the breast to ensure good contact between the 3D ABUS device and the skin. Then a curved panel (probe) was placed over the breast and gently flattened the tissue against the body. Images were acquired using a GE Invenia ABUS system (GE Healthcare, USA) which is equipped with $14 \mathrm{MHz}$ linear transducer.
The scan was performed with the patient in a supine position, a rolled towel is placed under the back of the patient on the side to be examined; this allows for flattening the breast for improved contact with the probe. After exposure of the breast, application of an adequate amount of ultrasound gel over the entire surface of the breast is done to ensure proper scanning and avoid artifacts. The transducer was applied to the breast using mild compression. Scanning was performed in three standard views (coronal, longitudinal, and transverse views) to allow for adequate coverage of the entire breast. Using the nipple as the center point, the anteroposterior (AP) position is first taken. Lateral position is taken by angling the probe from the axilla toward the sternum, and for medial position the probe is angled from the sternum toward the axilla. The scans are initially reviewed on the machine monitor for quality assurance of the acquired scan. The scan time for each acquisition was $1 \mathrm{~min}$ and volumetric data obtained were automatically transferred to the Invenia ABUS workstation for post processing including axial, coronal, and sagittal reformats and analysis. The entire examination time was approximately 15 min for both sides.

Ultrasound studies were reviewed for the presence or absence of lesions, location, size, number, outline, and contour (regular or irregular), margin or borders (micro or macro lobulations), echogenicity compared to the breast fat, and internal echo pattern (homogenous or heterogeneous).

\section{Image analysis}

The tomosynthesis and automated ultrasound data were evaluated separately by two experienced radiologists in consensus; both observers were unaware of the pathological data of each patient.

\section{Tomosynthesis (DBT) images}

Assessment of breast composition, mass characterization (shape, margin, density, multiplicity, location), asymmetry, calcification, axillary lymphadenopathy, extent of disease (EOD), skin thickening, retraction and architectural distortion, and BIRADS classification were also done.

\section{Automated ultrasound (ABUS) images}

The key descriptors that are used with ABUS to differentiate benign from malignant lesions are to evaluate the margin, echogenicity, posterior acoustic features, and parallel orientation of the lesion. Other signs used for ABUS included "white wall sign" which is the presence of an echogenic wall. It corresponds to acoustic enhancement found on US and suggests that the lesion is benign. On the coronal plane, a stellate lesion with desmoplastic retraction may appear as "a retraction 
phenomenon sign" being highly suspicious for malignancy.

Assessment of breast composition, mass characterization (shape, margin orientation, echo pattern, and posterior feature, multiplicity, location), calcification, axillary lymphadenopathy, extent of disease (EOD), skin thickening, retraction, and BIRADS classification were also done.

All breast masses included in this study were interpreted as above described and then the accuracy in reaching the final diagnosis was calculated for both tomosynthesis and automated ultrasound.

\section{Statistical analysis}

Data were coded and entered using the statistical package SPSS (Statistical Package for the Social Sciences) version 24. Data were summarized using frequency (count) and relative frequency (percentage) for categorical data. Standard diagnostic indices including sensitivity, specificity, positive predictive value (PPV), and negative predictive value (NPV) were calculated. For comparing categorical data, chi-square test was performed. Exact test was used instead when the expected frequency is less than $5, P$ value less than 0.05 was considered as statistically significant.

\section{Results}

This retrospective study included 70 female patients presented to the breast imaging unit with breast lesions (detected by mammographic examination or ultrasound, their mean age was $45 \pm 9 \mathrm{SD})$. Out of 70 patients, 82 masses were detected (12 cases showed bilateral breast lesions; 5 multifocal malignancies, 5 fibrocystic changes, and 2 bilateral fibroadenomas). Fifty-three cases (64.6\%) were diagnosed as benign while $29(35.3 \%)$ cases were diagnosed as malignant. As regarding breast density, 72 $(87.8 \%)$ of the examined breasts were ACR C, while 10 (12.2\%) were ACR D.

Pathological results were used as the gold standard of reference in 54 lesions (54/82): 30 core biopsies (USguided core needle biopsy), 19 malignant lesions, and 11 fibroadenomas. There were 11 surgical biopsies (10 malignant, 5 of which were multifocal and one benign angiolipoma) and 13 cases diagnosed on FNAC (2 fibroadenomas, 1 angiolipoma, and 10 fibrocystic changes). The remaining 28 lesions (28/82) were proven by US criteria to be benign (seven diagnosed as fibroadenomas (showing stationary course on 1 year follow-up), one fat containing lesion (angiolipoma with typical similar lesions at the back and extremities) and 20 were typical simple cysts on ultrasound) (Tables 1 and 2).

As regards the final BIRADS score given for both modalities, tomosynthesis showed accuracy of $93.1 \%$ in characterization of malignant masses with accuracy of
Table 1 Final pathological diagnosis of cases included in this study

\begin{tabular}{lll}
\hline Final diagnosis & Cases & \\
\cline { 2 - 3 } & Number & $\%$ \\
\hline IDC+/-DCIS & 22 & 26.8 \\
Metastasis from NEC & 1 & 2.4 \\
Metastatic melanoma & 1 & \\
Bilateral multifocal carcinoma & 5 & 6.1 \\
Angiolipoma & 3 & 3.6 \\
FCC & 28 & 34.1 \\
Fibroadenoma & 20 & 24.4 \\
Chronic galactocele & 2 & 2.4 \\
& Total & \\
& 82 & 100 \\
\hline
\end{tabular}

94.3\% in benign masses, on the other hand automated ultrasound showed 100\% accuracy in characterization of malignant masses with $98.1 \%$ accuracy in benign masses (Tables 3, 4, 5).

\section{Discussion}

Digital breast tomosynthesis is of interest for breast screening and diagnosis because of its 3D imaging capabilities and it is potential to alleviate a limitation of mammography, the masking of non-calcified cancers by other dense tissue [6]. Digital breast tomosynthesis has shown clinical potential compared to screen-film and digital mammography by subjectively providing better image quality and increasing the number of detected abnormalities and the accuracy in classification of masses [7].

The current research is a retrospective study on $70 \mathrm{pa}-$ tients presenting with dense breast masses diagnosed by mammographic examination. They have been evaluated by means of digital breast tomosynthesis and automated breast ultrasound, 82 masses were detected in the $70 \mathrm{pa}$ tients, pathological confirmation was done in most masses $(n=54 / 82)$ and ultrasonography confirmation in

Table 2 Method of reaching final diagnosis of cases included in this study

\begin{tabular}{lll}
\hline Method of diagnosis & $\begin{array}{l}\text { Number or } \\
\text { cases }\end{array}$ & Percentage \\
\hline Core biopsy & 30 & 36.5 \\
Surgical biopsy & 11 & 13.4 \\
FNA & 13 & 15.8 \\
$\begin{array}{l}\text { Typical imaging findings and 1-year } \\
\text { follow-up }\end{array}$ & 28 & 34.1 \\
& & \\
& Total & 100 \\
\hline
\end{tabular}


Table 3 Correlation between DBT results and pathology

\begin{tabular}{lll}
\hline DBT & Pathology & Malignant \\
\cline { 2 - 3 } & Benign & 2 \\
\hline Benign & 50 & 27 \\
Malignant & 3 & 29 \\
Total & 53 & \\
\hline
\end{tabular}

True positive, $27 / 29=93.1 \%$ (cases diagnosed malignant by both DBT and pathology)

False positive, $3 / 53=5.7 \%$ (cases diagnosed as malignant by DBT yet proved benign on pathology)

True negative, $50 / 53=94.3 \%$ (cases diagnosed benign by both DBT and pathology)

False negative, $2 / 29=6.9 \%$ (cases diagnosed as benign by DBT yet proved malignant on pathology)

the rest $(n=28 / 82)$; simple cysts, fat containing lesion (angiolipoma) and fibroadenomas.

Eighty-two masses were detected; among which 53 were benign $(n=53 / 82)$ and 29 were malignant $(n=29 /$ 82).

Histopathology of the masses was reached using different techniques such as core biopsy $(n=30)$, FNAC $(n=$ $13)$, and excisional biopsy $(n=11)$. The rest of the masses $(n=28 / 82)$ were confirmed by their characteristic sonographic appearances: anechoic simple cysts and mixed echogenicity angiolipoma.

In the current study, we found 2 cases of circumscribed masses as seen on DBT, yet ABUS revealed marginal micro lobulations. US-guided core needle biopsy revealed metastatic disease; one from a peritoneal carcinoid, neuroendocrinal tumor (NET) (Fig. 1), and the other case was a malignant melanoma metastasis. These cases were diagnosed as benign on DBT as the masses were well circumscribed and the margins were smooth; however, ultrasound revealed deep micro lobulations with posterior acoustic shadowing warranting biopsy. These two cases were considered as false negative by DBT.

Similar findings of false negative were reported by $\mathrm{Ki}$ $\mathrm{m}$ et al. in 2015 [8] where cases of lobular carcinoma appeared circumscribed on mammography. In another

Table 4 Correlation between ABUS results and pathology

\begin{tabular}{lll}
\hline ABUS & Pathology & \\
\cline { 2 - 3 } & Benign & Malignant \\
\hline Benign & 52 & 0 \\
Malignant & 1 & 29 \\
Total & 53 & 29 \\
\hline
\end{tabular}

True positive, $29 / 29=100 \%$ (cases diagnosed malignant by both ABUS and pathology)

False positive, $1 / 53=1.9 \%$ (cases diagnosed as malignant by ABUS yet proved benign on pathology)

True negative, $52 / 53=98.1 \%$ (cases proven negative by both ABUS and pathology)

False negative, $0 / 29=0 \%$ (cases diagnosed as benign by ABUS yet proved malignant on pathology)
Table 5 Comparison between accuracy measures of tomosynthesis and ABUS

\begin{tabular}{lll}
\hline Statistic & Tomosynthesis & ABUS \\
\hline Sensitivity & $93.1 \%$ & $100.0 \%$ \\
Specificity & $94.3 \%$ & $98.1 \%$ \\
Positive predictive value & $90.0 \%$ & $100.0 \%$ \\
Negative predictive value & $96.1 \%$ & $98.1 \%$ \\
Accuracy & $93.9 \%$ & $98.8 \%$ \\
\hline
\end{tabular}

study by Vaidya $\mathrm{T}$ et al. [9], metastatic breast lesions were seen well circumscribed and did not present with suspicious imaging features and was diagnosed purely on histopathology.

In the three cases of angiolipoma, DBT was easier to show the fatty content of the lesion; however, ABUS showed the heterogeneous echogenicity. One of these cases was surgically excised. The three patients had multiple bilateral angiolipomas with others in the back and extremities (Fig. 2).

Three cases were false positive on DBT appearing irregular shape with related architectural distortion proved to be two cases of bilateral chronic galactoceles/ abscesses and one case of inspissated secretions and periductal mastitis (chronic inflammatory changes) confirmed by fine needle aspiration. These were better diagnosed on ultrasound showing secretions within the ducts (Fig. 3).

One case of likely fibroadenoma as per DBT with wellcircumscribed margin showed deep micro lobulations with microcystic changes within the mass diagnosed as suspicious by ultrasound. Biopsy revealed a complex fibroadenoma; considered to be a false positive result by ABUS. Here, although DBT was true negative for malignancy, ultrasound added the cystic nature of the lesion.

Tomosynthesis in addition to ABUS showed favorable clinical trials and proved beneficial in many studies in the literature; similar to our study is the study of Larson et al. [10], which was performed in New York (USA) on 13 patients to evaluate a combined automated breast ultrasound and tomosynthesis in dense breast, it showed no obvious improvements made concerning the DBT alone; however, DBT did miss two benign masses because of high breast density. However, ABUS would have improved detection and be better able to catch masses that are missed by DBT for such reasons.

Another recent study by Abd Elkhalek et al. [11] concluded that the sensitivity of the ABUS is about $100 \%$, and that means, in all the results of the mammogram study, ABUS can detect it without significant change, while the specificity of the ABUS was about $62 \%$ and this was more evident in benign lesions. On the other hand, a study by Lee et al. in 2019 [7] found that adding ABUS to DBT and/or FFDM increased patient recall-rate in 

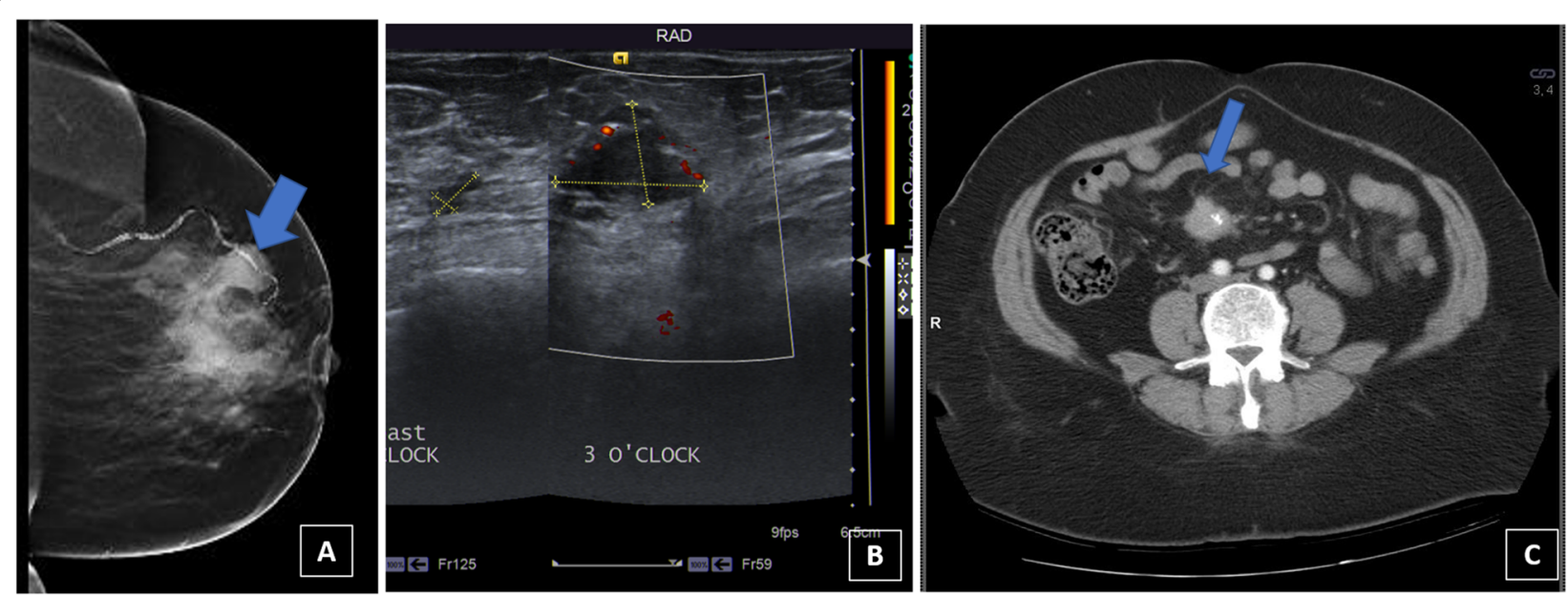

Fig. 1 Case one: 72-year-old female with left breast lump. A Left breast CC Tomo view showing a well circumscribed mass at 3 o'clock position (arrow). B HHUS with Doppler showing internal vascularity with an anterior advancing edge. ABUS showed a circumscribed hypoechoic mass (not shown). Core biopsy revealed neuroendocrinal carcinoma metastasis. C Axial CT slice of the lower abdomen showing the primary mesenteric carcinoid with central calcification and marginal speculation (arrow)

case of screening. It has been suggested in this study that double reading of ABUS images might reduce false positive recall rates. This might also be related to the fact that all patients included in this study had a positive family history or past personal history of breast cancer leading to a sampling bias. In another study by Tagliafico et al. in 2016 [12], they found that most of the cancers detected by ultrasound but not by tomosynthesis were masses, while the only cancer detected by tomosynthesis but not by ultrasound was an architectural distortion. In contrary to our study, patients with architectural distortion on mammography were seen as an area of hypoechoic architectural distortion on ultrasound with back-shadowing and were confirmed on core biopsy.

We had 5 cases of multifocal breast malignancy. Extent of disease (EOD) was more accurate on ABUS when correlated with surgical specimen (Fig. 4). This also coincides with Shin HJ in 2015 [13] who found that ABUS was more accurate to find EOD in cases of DCIS as compared to a second look handheld US. In summary, we found out that sensitivity of tomosynthesis in detection and characterization of breast masses was $93.1 \%$, the specificity was $94.3 \%$, the positive predictive value was $90 \%$, and the negative predictive value was $96.1 \%$. On the other hand, the sensitivity of automated
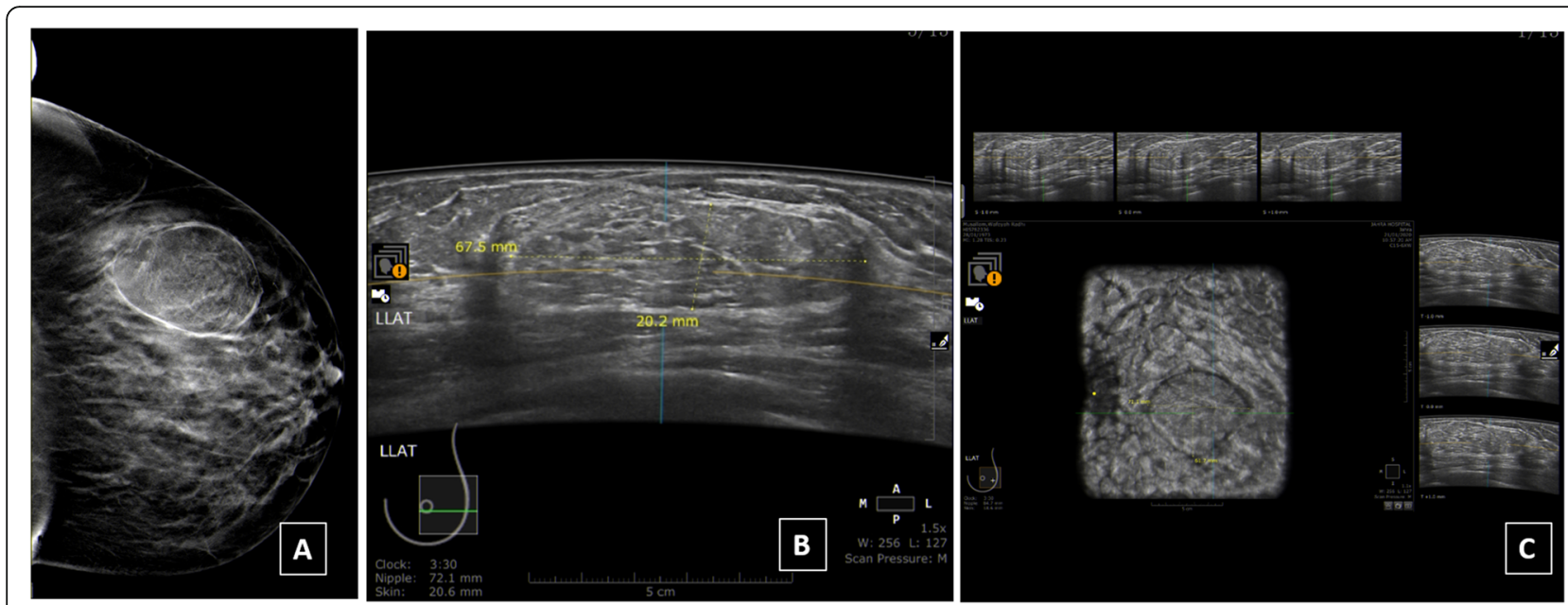

Fig. 2 Case two: 45-year-old female with enlarging left breast. A Tomo CC slice showing a well-defined lucent mass with a thick capsule (arrow). B, C ABUS images in transverse and multiplanar views showing the mass to be isoechoic with internal echogenicities and a surrounding echogenic capsule. Surgical excision revealed angiolipoma 


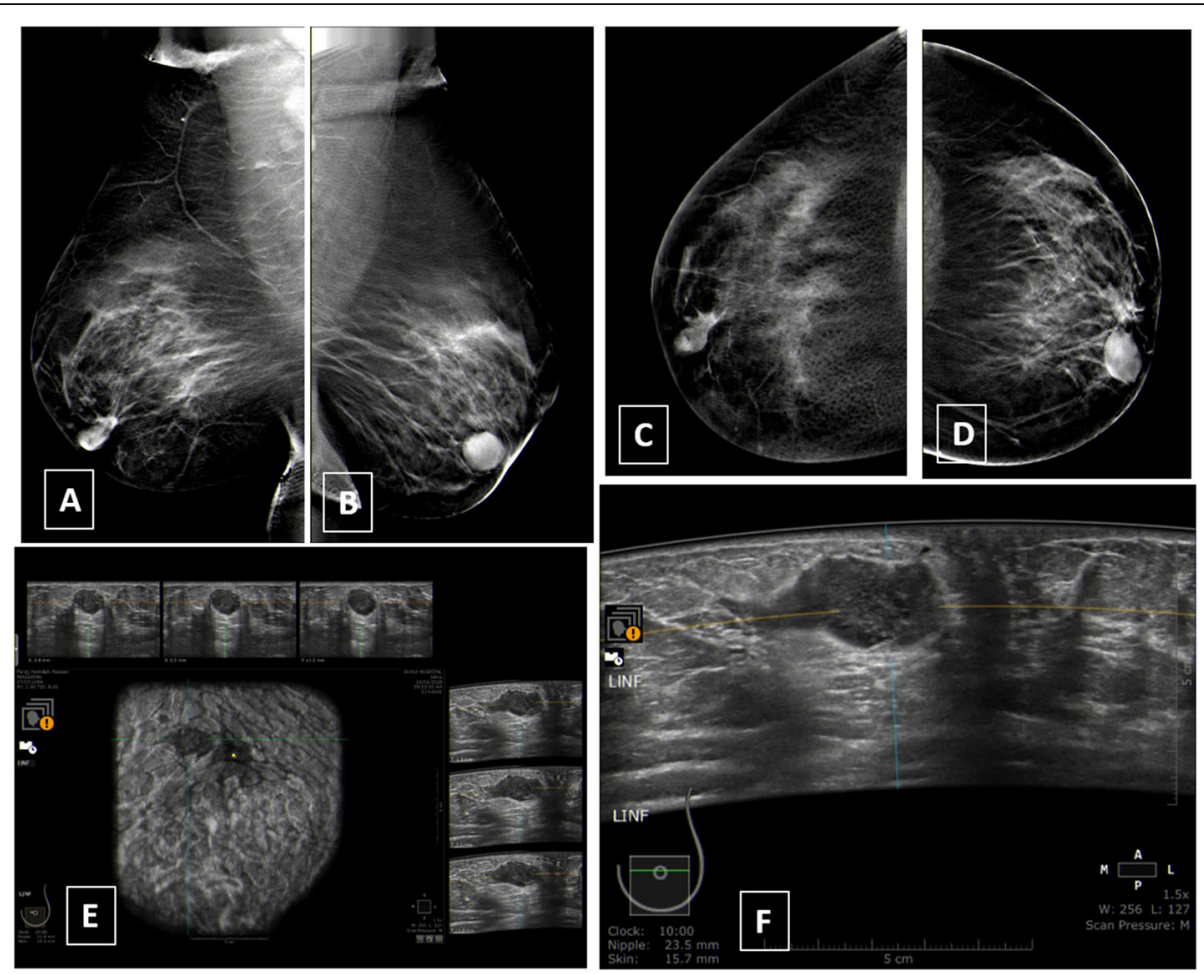

Fig. 3 Case three: 39-year-old with left breast lump. Recent history of right breast abscess drainage. Youngest child 18 months old. A-D 3D Tomo slices of both breasts in MLO and CC views showing bilateral retro areolar densities with partially obscured margins. E, F Multiplanar ABUS images showing cystic nature of the masses with intraductal echogenic secretions. Chronic galactocele

ultrasound was $100 \%$, the specificity was $98.1 \%$, the positive predictive value was $100 \%$, and the negative predictive value was $98.1 \%$.

\section{Conclusion}

Adding ABUS to tomosynthesis has proven a valuable imaging tool for characterization of breast lesions in dense breasts both as screening and diagnostic tool. In some cases of screening, it reduces patient call-back.
They can detect breast lesions whether malignant or benign and provide a higher diagnostic quality images with greater accuracy compared to digital mammography alone, especially in dense breast which is the greatest obstacle in breast imaging. They proved to be more sensitive and specific than digital mammography alone in showing tissue overlap, tumor characterization, lesion margins, extent of disease, and multiplicity of malignant lesions.

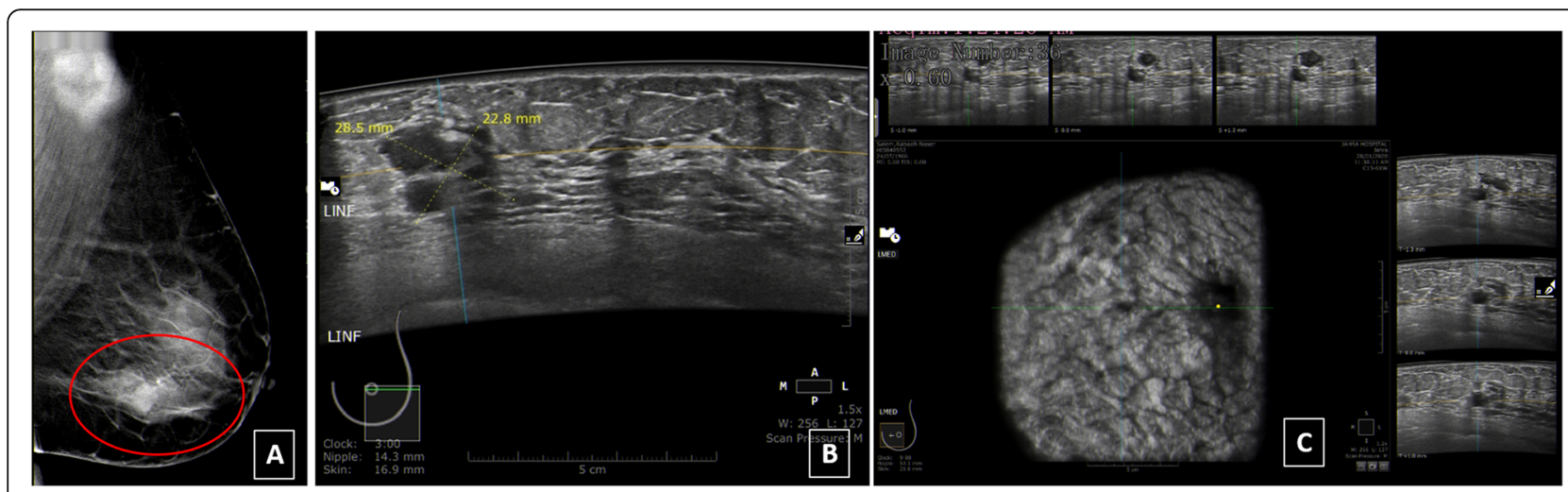

Fig. 4 Case four: 55-year-old female with left breast lump A Tomo MLO slice showing a dense spiculate mass with obscured margins (circle). B, C ABUS images in multiplanar views showing the mass to be multifocal hypoechoic with ill-defined margins. US-guided core biopsy revealed multifocal IDC with DCIS 


\section{Abbreviations}

ABUS: Automated breast ultrasound; AWBU: Automated 3D-ultrasound imaging of the whole breast; CC: Craniocaudal; DBT: Digital breast tomosynthesis; DBT: Digital breast tomosynthesis; DCIS: Duct carcinoma in situ; EOD: Extent of disease; HHUS: Handheld ultrasound; IDC: Invasive duct carcinoma; MLO: Medio-lateral oblique view; NET: Neuroendocrinal tumor

\section{Acknowledgements}

Thanks are due to patients, radiographers, and histopathologists who contributed to this work.

\section{Authors' contributions}

Equal sharing in data collection and analysis, manuscript writing, and editing by the three authors. OM and EA did the initial manuscript writing and data analysis. $\mathrm{RH}$ shared in writing, editing, and revising data and manuscript. All authors have read and approved the final manuscript.

\section{Funding}

Self-funding.

\section{Availability of data and materials}

All data and materials used in this research are available.

\section{Declarations}

\section{Ethics approval and consent to participate}

The study protocol was approved by the Local Ethics Committee. Vancouver ethical standards were followed in the conduct of the study. No available ethics' committee reference number. All patients signed a written consent to perform the procedure.

\section{Consent for publication}

All patients included in this research gave written informed consent to publish the data contained within this study.

\section{Competing interests}

The authors declare they have no competing interests.

\section{Author details}

${ }^{1}$ Clinical and Interventional Radiology, faculty of Medicine, Cairo University, Cairo, Egypt. ${ }^{2}$ Radiology, National Cancer Institute, Cairo University, Cairo, Egypt.

Received: 31 December 2020 Accepted: 7 July 2021

Published online: 12 August 2021

\section{References}

1. Vourtsis A, Berg WA (2019) Breast density implications and supplemental screening. Eur Radiol 29(4):1762-1777. https://doi.org/10.1007/s00330-0185668-8

2. Kopans DB (2014) Digital breast tomosynthesis from concept to clinical care. Am J Roentgenol 202(2):299-308. https://doi.org/10.2214/AJR.13.11520

3. Ali EA, Adel $L$ (2019) Study of role of digital breast tomosynthesis over digital mammography in the assessment of BIRADS 3 breast lesions. Egypt J Radiol Nucl Med 50(1):48. https://doi.org/10.1186/s43055-019-0052-5

4. Zanotel M, Bednarova I, Londero V, Linda A, Lorenzon M, Girometti R, Zuiani C (2018) Automated breast ultrasound: basic principles and emerging clinical applications. Radiol Med. 123(1):1-12. https://doi.org/10.1007/s11547017-0805-z Epub 2017 Aug 28. PMID: 28849324

5. Brem R, Tabár L, Duffy S, Inciardi M, Guingrich J, Hashimoto B et al (2015) Assessing improvement in detection of breast cancer with threedimensional automated breast US in women with dense breast tissue: the Somolnsight study. Radiology 274(3):663-673. https://doi.org/10.1148/ra diol.14132832

6. Heil J, Barr RG, Radicke M et al (2018) Initial results of the FUSION-X-US prototype combining 3D automated breast ultrasound and digital breast tomosynthesis. Eur Radiol 28(6):2499

7. Lee JM, Partridge SC, Liao GJ, Hippe DS, Kim AE, Lee Cl, Rahbar H, Scheel JR, Lehman CD (2019) Double reading of automated breast ultrasound with digital mammography or digital breast tomosynthesis for breast cancer screening. Clin Imaging 55:119-125. https://doi.org/10.1016/j.clinimag.2019. 01.019

8. Kim SA, Chang JM, Cho N, Yi A, Moon WK (2015) Characterization of breast lesions: comparison of digital breast tomosynthesis and ultrasonography. Korean J Radiol 16(2):229-238. https://doi.org/10.3348/kjr.2015.16.2.229

9. Vaidya T, Ramani S, Rastogi A (2018) A case series of metastases to the breast from extramammary malignancies. Indian J Radiol Imaging 28(4):470475. https://doi.org/10.4103/ijri.JJRI_218_18

10. Larson ED, Lee W-M, Roubidoux MA, Goodsitt MM, Lashbrook C, Davis CE, Carson PL (2018) Preliminary clinical experience with a combined automated breast ultrasound and digital breast tomosynthesis system. Ultrasound Med Biol 44(3):734-742. https://doi.org/10.1016/j.ultrasmedbio.2 017.12 .003

11. Abd Elkhalek Yl, Bassiouny AM, Hamid RWARA (2019) Automated breast ultrasound system (ABUS): can it replace mammography as a screening tool? Egypt J Radiol Nucl Med 50(1):51. https://doi.org/10.1186/s43055-0190051-6

12. Tagliafico AS, Calabrese M, Mariscotti G, Manuela D, Tosto S, Monetti F, Airaldi S, Bignotti B, Nori J, Bagni A, Signori A, Sormani MP, Houssami N (2016) Adjunct screening with tomosynthesis or ultrasound in women with mammography-negative dense breasts: interim report of a prospective comparative trial. J Clin Oncol 34(16):1882-1888. https://doi.org/10.1200/ JCO.2015.63.4147

13. Shin HJ, Kim HH, Cha JH (2015) Current status of automated breast ultrasonography. Ultrasonography. 34(3):165-172. https://doi.org/10.14366/ usg.15002

\section{Publisher's Note}

Springer Nature remains neutral with regard to jurisdictional claims in published maps and institutional affiliations.

\section{Submit your manuscript to a SpringerOpen ${ }^{\circ}$ journal and benefit from:}

- Convenient online submission

- Rigorous peer review

- Open access: articles freely available online

High visibility within the field

- Retaining the copyright to your article

Submit your next manuscript at $\boldsymbol{\nabla}$ springeropen.com 